\title{
Cholangiocarcinoma in Sri Lanka :experience of a tertiary referral centre
}

\author{
H S Perera ${ }^{1}$, R G M S Nandasena ${ }^{1}$, M D Jayawardene ${ }^{2}$, B D Chandragupta ${ }^{1}$, S Piyarathne $^{1}$, A A Pathirana ${ }^{1}$ \\ ${ }^{1}$ Department of Surgery, Faculty of Medical Sciences, University of Sri Jayewardenepura \\ ${ }^{2}$ Professorial Surgical Unit, Colombo South Teaching Hospital, Kalubowila
}

Keywords: Cholangiocarcinoma; CA19-9; resectability; operability

\begin{abstract}
\section{Introduction}

Cholangiocarcinoma is a malignancy arising from the epithelial lining of the biliary tree, which is associated with a poor outcome.
\end{abstract}

\section{Objectives}

To describe the relative incidence of each type of cholangiocarcinoma, gender distribution, common presenting symptoms, the prevalence of metastatic disease and assess the resectability rates and to assess the relationship of different types of cholangiocarcinoma with CA19-9 levels.

Methods: A descriptive cross-sectional study, of patients diagnosed with cholangiocarcinoma, whose details were obtained from the hepato-pancreato-biliary database that is maintained in a tertiary care unit, in Sri Lanka. Quantitative variables expressed as the mean and standard deviation (SD) and qualitative variables expressed in percentages. The statistical analyses were carried out using SPSS version 25 with statistical significance defined as $\mathrm{P}<0.05$.

\section{Results}

One hundred and twenty patients $(n=120)$ were studied. There was a higher incidence of cholangiocarcinoma among males. Average age of presentation was 61.8 years, with males presenting at a slightly older age. Hilar cholangiocarcinomas were the most common type, followed by distal and intrahepatic. Jaundice was the most common presenting symptom among distal and hilar cholangiocarcinomas. CA 19-9 levels were found to be elevated in $71.9 \%$ of the patients. Patients with hilar and intrahepatic cholangiocarcinomas had a higher incidence of advanced disease at the time of presentation with relatively low resectability rates observed among these patients.

\footnotetext{
Correspondence: H S Perera

E-mail: sahan_doc@yahoo.com

(iD) https://orcid.org/0000-0003-3973-1292

Received: 11-01-2021 Accepted: 30-03-2021
}

DOI: http://doi.org/10.4038/sljs.v39i1.8792

\section{Discussion}

The demographic characteristics and the relative incidence of each type of cholangiocarcinoma was similar to those observed in the western world. However, we noted that the resectability rates in our study to be significantly lower in comparison.

\section{Introduction}

Cholangiocarcinoma is a malignancy arising from the epithelial lining of the biliary tree. Traditionally cholangiocarcinomas were classified as intrahepatic and extrahepatic tumours, with those arising proximal to the second order bile ducts being denoted as intrahepatic tumours. However, contemporary studies have shown that perihilar and distal cholangiocarcinomas which are separated at the point of insertion of the cystic duct, are unique in tumour biology [1], which has led to them being identified as distinct entities with individual staging, management protocols and prognostic parameters. Majority of the cholangiocarcinomas are adenocarcinomas, other histological types are also rarely seen.

While surgery remains the only potentially curative therapy, most patients are deemed to be unresectable due to advanced stage of the disease at the time of presentation [1], thus making cholangiocarcinoma a disease with poor outcome and high mortality. Traditionally a higher incidence has been noted among Asian populations as opposed to Europeans and North Americans [1]. Although the overall incidence is low in comparison to other malignancies, studies have shown that the incidence of cholangiocarcinoma, especially intrahepatic cholangiocarcinoma is steadily rising [2][3]. Intrahepatic cholangiocarcinoma is the second commonest primary hepatic malignancy following hepatocellular carcinoma [4], thus making it an important differential diagnosis which needs to be excluded in patients presenting with a malignant liver lesion.

Primary sclerosing cholangitis, inflammatory bowel disease, hepatolithiasis, choledochal cysts and biliary parasites are some of the recognized risk factors of cholangiocarcinoma, while smoking and alcohol are up and coming risk factors [5][6]. Recent studies have suggested hepatitis B \& C, diabetes mellitus and obesity as potential risk factors [5][6]. 
However, there is insufficient evidence to support this.

To date there has not been any studies describing the demographic and clinical characteristics of cholangiocarcinoma in Sri Lanka. While such a study is long overdue, it will not only provide as a source for surgical units in Sri Lanka to better understand cholangiocarcinoma, but also would provide a platform to build on and carry out further extensive studies, thus uplifting the standard of health care provision and benefiting the patients with cholangiocarcinoma in turn.

\section{Materials and Method}

The aim of this study was to describe the relative incidence of each type of cholangiocarcinoma, gender distribution, common presenting symptoms, the prevalence of metastatic disease and assess the resectability rates. The relationship between the CA19-9 levels with each subset of cholangiocarcinoma was also evaluated. CA19-9 level $>100$ $\mathrm{U} / \mathrm{L}$ were more likely to have malignant aetiology and were considered as elevated for this study[7]. However, not all patients with extrahepatic cholangiocarcinomas had their biliary system decompressed prior to CA19-9 measurement, which was identified as a limitation.

Patients with cholangiocarcinoma, almost all of whom were diagnosed from January 2016 to March 2020, at a hepatopancreato-biliary multidisciplinary team meeting in the presence of surgeons, radiologists, and an oncologist. Histological diagnosis was available only in those who underwent surgery and those who needed biopsy due to diagnostic doubt or when histology was needed prior to palliative chemotherapy. In majority the diagnosis was made radiologically with the aid of CECT and MRI/MRCP. This was identified as a limitation in the study. The characteristic CECT findings of intrahepatic Cholangiocarcinomas, capsular retraction, dilated bile ducts distal to the mass and delayed tumoral enhancement were used to distinguish it from hepatocellularcarcinoma. Details were obtained from the hepato-pancreato-biliary database that is maintained in the unit. While our study was restricted to patients presenting to and are followed up at our unit, it is important to note that our database comprised of patients from 13 districts (out of 25) in the country with majority from the Colombo (42\%) and Kalutara (19\%) districts.

Most of our study is descriptive in nature, with quantitative variables expressed as the mean and standard deviation (SD) and qualitative variables expressed in percentages. The statistical analyses were carried out using SPSS version 25 with statistical significance defined as $\mathrm{P}<0.05$.

\section{Results}

There were 120 patients. Males accounted for $55 \%$ of the study population. The average age of presentation of cholangiocarcinoma was 61.8 years, ranging from 29 to 87 . The average age of the male patients was 63 years, while that of the female patients was 60 years. The average age of presentation of distal, hilar and intra hepatic cholangiocarcinomas were $65.2,61.4$ and 57.2 years respectively.

Male patients accounted for $52.6 \%, 60.3 \%$ and $48 \%$ of distal, hilar and intrahepatic cholangiocarcinomas respectively.

Table 1. Patient characteristics

\begin{tabular}{|l|l|l|l|}
\hline & $\begin{array}{l}\text { Distal } \\
(\mathbf{n = 3 9 )}\end{array}$ & $\begin{array}{l}\text { Hilar } \\
(\mathbf{n = 5 5 )}\end{array}$ & $\begin{array}{l}\text { Intrahepatic } \\
(\mathbf{n = 2 6 )}\end{array}$ \\
\hline Average age (vears) & 65.2 & 61.4 & 57.2 \\
\hline Male (\%) & 52.6 & 60.3 & 48 \\
\hline Female(\%) & 47.3 & 39.6 & 52 \\
\hline
\end{tabular}

The relative incidence of distal, hilar and intrahepatic cholangiocarcinoma was $32.5 \%, 45.8 \%$ and $21.7 \%$ respectively. Of the hilar cholangiocarcinomas Bismuth Corlette type 2 accounted for $50 \%$ of the cases with types $3 a$, $3 \mathrm{~b}$ and 4 accounting for the rest.

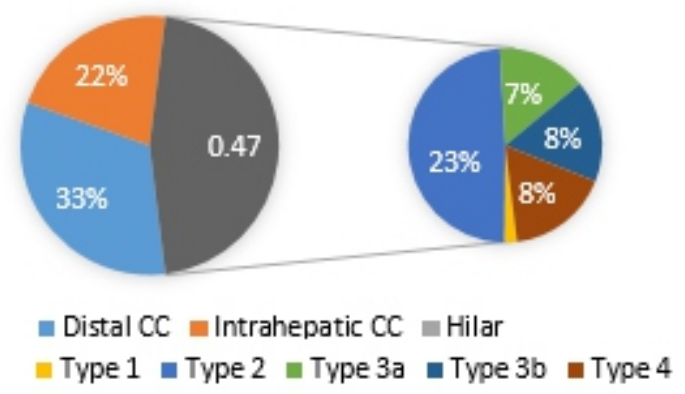

Figure 1. Incidence of each type of cholangiocarcinoma

Jaundice was the presenting symptom of majority of distal and hilar cholangiocarcinoma patients, $85 \%$ and $72 \%$ respectively. Less than $20 \%$ of the patients with intrahepatic cholangiocarcinomas were jaundiced at the time of presentation. Among patients with intra hepatic cholangiocarcinoma the most common presenting symptom was abdominal pain seen in $34.6 \%$ of the patients.

Table 2. Presenting symptoms

\begin{tabular}{|l|c|c|c|c|}
\hline Symptom & $\begin{array}{l}\text { Distal } \\
(\mathbf{n}=\mathbf{3 9})\end{array}$ & $\begin{array}{l}\text { Hilar } \\
(\mathbf{n}=\mathbf{5 5})\end{array}$ & $\begin{array}{l}\text { Intrahepatic } \\
(\mathbf{n}=\mathbf{2 6})\end{array}$ & $\begin{array}{l}\text { Total } \\
(\mathbf{n}=\mathbf{1 2 0})\end{array}$ \\
\hline Jaundice & 33 & 40 & 5 & 78 \\
\hline Abdominal pain & 0 & 0 & 9 & 9 \\
\hline $\begin{array}{l}\text { Loss of appetite and } \\
\text { weight }\end{array}$ & 13 & 16 & 7 & 36 \\
\hline Fever/cholangitis & 5 & 10 & 0 & 15 \\
\hline incidental & 0 & 2 & 5 & 7 \\
\hline
\end{tabular}

The Sri Lanka Journal of Surgery 2021; 39(1): 01-04 
CA 19-9 levels were found to be elevated in $71.9 \%$ of the patients with cholangiocacinoma. Elevated CA 19-9 levels were seen in $75 \%, 71 \%$ and $66.6 \%$ of the patients with intrahepatic, hilar and distal cholangiocarcinomas respectively.

The proportion of patients found to have metastatic disease at the time of presentation was $21 \%, 33 \%$ and $35 \%$ for distal, hilar and intrahepatic cholangiocarcinomas respectively.

Table 3. Metastatic disease

\begin{tabular}{|l|c|c|c|}
\hline & $\begin{array}{c}\text { Distal } \\
(\mathbf{n}=\mathbf{8})\end{array}$ & $\begin{array}{c}\text { Hilar } \\
(\mathbf{n}=\mathbf{1 8})\end{array}$ & $\begin{array}{c}\text { Intrahepatic } \\
(\mathbf{n}=\mathbf{9})\end{array}$ \\
\hline Liver & 3 & 12 & 0 \\
\hline Lung & 2 & 2 & 4 \\
\hline $\begin{array}{l}\text { Non-regional } \\
\text { lymph nodes }\end{array}$ & 1 & 2 & 3 \\
\hline Peritoneal & 2 & 2 & 2 \\
\hline
\end{tabular}

Of the patients with distal cholangiocarcinoma $(n=39), 61.5 \%$ had resectable disease $(n=24)$ at the time of presentation. However, only $50 \%(n=12)$ of these patients were deemed fit for surgery. Twelve patients underwent pancreatoduodenectomy and twenty-five patients underwent palliative metal stenting during this period.

Among the patients with hilar cholangiocarcinoma only $20 \%$ had resectable disease at the time of presentation. Only $27 \%$ of the patients with resectable disease were fit enough for surgery with two left and one right hepatectomy being performed in this period. Thirty-seven patients underwent palliative metal stenting.

Thirty-two percent $(32 \%)$ of the patients with intrahepatic cholangiocarcinoma had disease amenable to resection at the time of presentation. All of them were deemed fit for curative therapy, with 2 left and 2 right hepatectomy, one nonanatomical liver resection and two locoregional therapy (1 TACE and 1 MWA) being performed. Eight patients were referred for palliative chemotherapy.

Table 4. Resectability and Operability

\begin{tabular}{|l|l|l|l|}
\hline & DCC $(\mathbf{n = 3 9 )}$ & HCC $(\mathbf{n = 5 5 )}$ & ICC $(\mathbf{n = 2 5 )}$ \\
\hline Unresectable & $8(20.5 \%)$ & $37(67.3 \%)$ & $13(52 \%)$ \\
\hline Indeterminate & $7(18 \%)$ & $7(12.7 \%)$ & $4(16 \%)$ \\
\hline Resectable & $24(61.5 \%)$ & $11(20 \%)$ & $8(32 \%)$ \\
\hline a) Operable & 12 & 3 & 8 \\
\hline b) Inoperable & 12 & 8 & 0 \\
\hline
\end{tabular}

\section{Discussion}

Males were the predominant gender among cholangiocarcinoma patients and they were found to present at an older age than females. Patients with distal and hilar cholangiocarcinomas were more likely to present at an older age in comparison to intra cholangiocarcinomas and this was statistically significant $(p=0.02)$. While the gender distribution of distal cholangiocarcinomas (male-52\%) and intrahepatic cholangiocarcinomas (male-48\%) were relatively similar, males were the predominant gender in hilar cholangiocarcinomas $(60 \%)$. However, this was not statistically significant $(\mathrm{p}=0.3)$. Hilar cholangiocarcinoma was the most common type of cholangiocarcinoma (45.8\%), followed by distal (32.5\%) and intrahepatic (21.7\%) cholangiocarcinomas.

Patients with distal and hilar cholangiocarcinomas were more likely to present with jaundice, while those with intrahepatic cholangiocarcinomas were more likely to present with abdominal pain. Both these associations were found to be statistically significant $(\mathrm{p}<0.05)$. In a study by Forner et. Al, it was described that the most characteristic and common symptom of extrahepatic cholangiocarcinomas is jaundice. In the case of intrahepatic cholangiocarcinomas, jaundice is the initial symptom only in around $10 \%-15 \%$ of the cases, when biliary obstruction would mainly be related to obstruction of the liver hilum by lymph nodes or migration of detritus and subsequent failure of the correct drainage of the biliary ducts. When Cholangiocarcinoma patients present with symptoms others than jaundice, they most frequently include abdominal pain, malaise, night sweats, asthenia, nausea and weight loss.

CA 19-9 tumour marker levels were elevated in $71.9 \%$ of patients with cholangiocarcinoma. It was noted that the more proximal the malignancy the higher the probability of an elevated CA 19-9 levels however no statistical correlation was found for this association. In our study $46 \%$ of IHCC, $25 \%$ distal and 30\% hilar cholangiocarcinoma had CA19-9 values more than 1000 .

Patients with hilar and intrahepatic cholangiocarcinomas were more likely to have advanced disease at the time of presentation and this is reflected in the relatively low resectability rates of these patients in comparison to distal cholangiocarcinoma.

As studies on cholangiocarcinoma are sparse in Sri Lanka and within the South Asian region, we compared the results of our study with that of a similar study carried out by Nakeeb A, et al at The Johns Hopkins Medical Institutions, Baltimore, Maryland [8]. The demographic characteristics and the relative incidence of each type of cholangiocarcinoma is similar in both studies. However, we noted that the 
Table 5. Our study compared to western published literature

\begin{tabular}{|c|l|l|}
\hline & Our study & $\begin{array}{l}\text { Nakeeb A, et al. } \\
\text { Ann Surg } \\
1996 ; 224: 463^{1} \\
\text { The Johns } \\
\text { Hopkins Medical } \\
\text { Institutions, USA) }\end{array}$ \\
\hline $\begin{array}{c}\text { Overall Average age } \\
\text { Age range }\end{array}$ & $\begin{array}{l}61.8 \text { years } \\
29-87 \text { years }\end{array}$ & $\begin{array}{l}62.2 \text { years } \\
23-84 \text { years }\end{array}$ \\
\hline $\begin{array}{c}\text { Average age } \\
\text { a) Distal } \\
\text { b) Hilar } \\
\text { c) Intrahepatic }\end{array}$ & 65 years & 65 years \\
\hline Male \% years & 52 years \\
\hline Resectability & $55 \%$ & $55 \%$ \\
a) Distal & $61.5 \%$ & $91 \%$ \\
b) Hilar & $20 \%$ & $56 \%$ \\
c) Intrahepatic & $32 \%$ & $50 \%$ \\
\hline
\end{tabular}

resectability rates in our study to be significantly lower in comparison.

While it is difficult to point out the exact reason for the low resectability rates in Sri Lanka in comparison to the western world, one can speculate that poor patient awareness of the disease, late presentation, inadequate resources (hence longer periods spent arriving at the diagnosis) and longer waiting lists as probable contributory factors.

\section{Conclusions}

Cholangiocarcinoma is a malignancy with poor outcomes and further studies need to be conducted at national level to ascertain its risk factors. There is a need to assess the survival rates of these patients and to determine the overall incidence of cholangiocarcinomas in comparison to other gastrointestinal malignancies. This will aid in broadening the knowledge of cholangiocarcinoma among the medical practitioners of Sri Lanka and will undoubtedly benefit the patients with this disease.
All authors disclose no conflict of interest. The study was conducted in accordance with the ethical standards of the relevant institutional or national ethics committee and the Helsinki Declaration of 1975, as revised in 2000 .

\section{References}

1. Boris Blechacz. Cholangiocarcinoma: Current Knowledge and New Developments. Gut and Liver, Vol. 11, No. 1, January 2017, pp. 13-26

2. Yasser Shaib, Hashem B. El-Serag. The Epidemiology of Cholangiocarcinoma. Seminars in liver disease/volume 24, number 22004

3. Kim Y, Moris DP, Zhang X-F, et al. Evaluation of the 8th edition American Joint Commission on Cancer (AJCC) staging system for patients with intrahepatic cholangiocarcinoma: A surveillance, epidemiology, and end results (SEER) analysis. J Surg Oncol. 2017;9999:1-8. https://doi.org/10.1002/jso.24720

4. Han Zhang, Tian Yang, Mengchao Wu, Feng Shen. Intrahepatic cholangiocarcinoma: epidemiology, risk factors, diagnosis and surgical management. Cancer Letters (2015). http://dx.doi.org/doi: 10.1016/j.canlet.2015.09.008.

5. Chinchilla-López P, Aguilar-Olivos NE, García-Gómez J, et al.. Prevalence, Risk Factors, and Survival of Patients with Intrahepatic Cholangiocarcinoma. Ann Hepatol. 2017 JulAug;16(4):565-568

6. Rizvi S, Gores GJ. Pathogenesis, diagnosis, and management of cholangiocarcinoma. Gastroenterology. 2013; 145:1215-1229. [PubMed: 24140396]

7. Saluja SS, Sharma R, Pal S, Sahni P, Chattopadhyay TK. Differentiation between benign and malignant hilar obstructions using laboratory and radiological investigations: a prospective study. HPB (Oxford). 2007;9(5):373-82. doi: 10.1080/13651820701504207. PMID: 18345322; PMCID: PMC2225516.

8. Attila Nakeeb, Henry A. Pitt, Taylor A. Sohn, et al. Cholangiocarcinoma A Spectrum of Intrahepatic, Perihilar, and Distal Tumors. ANNALS OF SURGERY Vol. 224, No. 4.463-475 